\title{
Erratum to: On the relation between pole and running heavy quark masses beyond the four-loop approximation
}

\author{
A. L. Kataev ${ }^{1,2, *}$ and V.S. Molokoedov ${ }^{1,2,3, * *}$ \\ ${ }^{1}$ Institute for Nuclear Research of the Russian Academy of Sciences, \\ 60th October Anniversary prospect 7a, Moscow, 117312, Russia \\ ${ }^{2}$ Moscow Institute of Physics and Technology, \\ Institusky per. 9, Dolgoprudny, Moscow region, 141700, Russia \\ ${ }^{3}$ Landau Institute for Theoretical Physics of the Academy of Sciences of Russia, \\ Akademika Semenova avenue 1-a, Chernogolovka, Moscow Region, 142432, Russia
}

\begin{abstract}
The effective charges motivated method is applied to the relation between pole and $\overline{\mathrm{MS}}$-scheme heavy quark masses to study high order perturbative QCD corrections in the observable quantities proportional to the running quark masses. The non-calculated five- and six-loop perturbative QCD coefficients are estimated. This approach predicts for these terms the sign-alternating expansion in powers of number of lighter flavors $n_{l}$, while the analyzed recently infrared renormalon asymptotic expressions do not reproduce the same behavior. We emphasize that coefficients of the quark mass relation contain proportional to $\pi^{2}$ effects, which result from analytical continuation from the Euclidean region, where the scales of the running masses and QCD coupling constant are initially fixed, to the Minkowskian region, where the pole masses and the running QCD parameters are determined. For the $t$-quark the asymptotic nature of the non-resummed PT mass relation does not manifest itself at six-loops, while for the $b$-quark the minimal PT term appears at the probed by direct calculations four-loop level. The recent infrared renormalon based studies support these conclusions.
\end{abstract}

Original article:

EPJ Web of Conferences 191, 04005 (2018),

XXth International Seminar on High Energy Physics (QUARKS-2018),

https://doi.org/10.1051/epjconf/201819104005

Citations in the original article are corrupted due to error made by Editors QUARKS-2018. Full text with correct citations follows.

\section{Introduction}

It is well-known that the masses of charm, bottom and top-quarks are one of the most important QCD parameters, which are relevant for processing different data, obtained at LHC and

\footnotetext{
*e-mail: kataev@ms2.inr.ac.ru

**e-mail: viktor_molokoedov@mail.ru
} 
Tevatron. The pole and $\overline{\mathrm{MS}}$-scheme running heavy quark masses are the generally accepted definitions for these parameters. The first ones are determined by the position of pole of the renormalized fermion propagator at Minkowskian region $k^{2}=M_{q}^{2}$. The scale-dependence of the second ones are defined from the solution of the following renormalization-group equation:

$$
\frac{\bar{m}_{q}(s)}{\bar{m}_{q}\left(\mu^{2}\right)}=\exp \left(\int_{a_{s}\left(\mu^{2}\right)}^{a_{s}(s)} \frac{\gamma_{m}(x)}{\beta(x)} d x\right) .
$$

Here $\bar{m}_{q}\left(\mu^{2}\right)$ are the running masses of heavy quarks, normalized at the Minkowskian scale $\mu^{2}, a_{s}\left(\mu^{2}\right)=\alpha_{s}\left(\mu^{2}\right) / \pi$ is the renormalized QCD coupling constant in the $\overline{\mathrm{MS}}$-scheme, $s$ is the energy time-like variable. The RG $\beta$-function and anomalous mass dimension $\gamma_{m}$ are defined as:

$$
\mu^{2} \frac{\partial a_{s}}{\partial \mu^{2}}=\beta\left(a_{s}\right)=-\sum_{n=0}^{\infty} \beta_{n} a_{s}^{n+2}, \quad \mu^{2} \frac{\partial \log \left(\bar{m}_{q}\right)}{\partial \mu^{2}}=\gamma_{m}\left(a_{s}\right)=-\sum_{n=0}^{\infty} \gamma_{n} a_{s}^{n+1} .
$$

Their five-loop approximations in the $\overline{\mathrm{MS}}$-scheme can be found in works of $[1,2]$ and [3] correspondingly. It is worth to emphasize that the renormalization scale $\mu^{2}$ in Eqs. $(1,2)$ may be initially defined in the Euclidean region. As was shown in [4-9] the pole quark mass relation is sensitive to the long-distance infrared renormalon (IRR) effects. This IRR sensitivity leads to the $O\left(\Lambda_{\mathrm{QCD}}\right)$ renormalon ambiguity in the determination of heavy quark masses, which is related to the first $\delta=1 / 2$ IRR pole in the $\delta$-plane of the Borel transform for the PT QCD relation between pole and running heavy quark masses [5]. Therefore, the significant attention is paid to the determinations of masses in the $\overline{\mathrm{MS}}$-scheme, which do not imply the need for knowledge of the long-distance contributions. Indeed, their definition within dimensional regularization presumes taking into account of the UV divergent poles only. Therefore sometimes the running $\overline{\mathrm{MS}}$ heavy quark masses are called the short-distance masses, which unlike pole masses have nothing in common with the IRR effects.

In view of all mentioned above it is of interest to consider the relation between pole and running masses of heavy quarks:

$$
M_{q}=\bar{m}_{q}\left(\bar{m}_{q}^{2}\right) \sum_{n=0}^{\infty} t_{n}^{M} a_{s}^{n}\left(\bar{m}_{q}^{2}\right)
$$

The choice $\mu^{2}=\bar{m}_{q}^{2}$ corresponds to the commonly accepted way of fixation of the renormalization scale in the Minkowskian region, wherein coefficients $t_{n}^{M}$ are polynomials in powers of the number of massless flavors $n_{l}$

$$
t_{n}^{M}=\sum_{k=0}^{n-1} t_{n k}^{M} n_{l}^{k}
$$

with the initial condition $t_{0}^{M}=1$. Note that we consider the approximation when heavy flavor number $n_{f}$ of active quarks are defined as $n_{f}=n_{l}+1$. The results of calculations of one, two and three-loop corrections to (3) were obtained in [10], [11-13], [14, 15] respectively. The four-loop contributions $t_{43}^{M}$ and $t_{42}^{M}$ are known from the analytical calculations, performed in [16]. The rest $O\left(a_{s}^{4}\right)$-corrections, namely $t_{41}^{M}$ and $t_{40}^{M}$-terms, are not yet computed in analytical form, but are evaluated numerically with the corresponding theoretical uncertainties. The total expressions for the $t_{4}^{M}$-term at fixed numbers of massless flavors $n_{l}=3,4,5$ were evaluated in [17]. Using these numerical results, supplemented with the analytical information on 
$t_{43}^{M}$ and $t_{42}^{M}$-terms, the authors of Ref.[18] presented the first estimates of the unknown previously $t_{41}^{M}$ and $t_{40}^{M}$-coefficients by means of the renormalon-inspired approach. Independently, the numerical values of these terms were determined in $[19,20]$ with the help of mathematically self-consistent least squares (LS) method, which is well-defined procedure of solving the overdetermined system of linear equations with the fixation of theoretical inaccuracies of the central values of the obtained results. Recently the updated results of the numerical evaluation of $t_{4}^{M}$-terms were obtained by means of the Monte-Carlo methods in [21] not for three values of $n_{l}$ only but at extra 18 values as well, which corresponds to the additional points in the studied interval $0 \leq n_{l} \leq 20$. The results of these updated computations were obtained with considerably smaller uncertainties than the ones, presented in [17]. This prompted us to reconsider the results of [19] and to get new values of $t_{41}^{M}, t_{40}^{M}$-terms and their uncertainties using the same LS method [22]. The central values of new results have changed slightly (see the Note added to [19] as well). This is related to slight change of the central values of the improved more precise numbers from Ref.[21]. Moreover, thanks to the increase of the number of analyzed by LS method equations, fixed by the results from [21], which have smaller numerical errors than the outcomes of previous calculations [17], the obtained in [22] solutions of larger system of linear equations turned out to be drastically more precise than the ones, obtained previously in Ref.[19]. This feature is in agreement with general property of the mathematical LS approach. Leaving the discussions of the technical issues of this method aside we will return now to the consideration of the asymptotic structure of Eq.(3).

Due to the manifestation of the IRRs in the Borel image for the pole-running heavy quark mass relation, it is possible to conclude that the mass conversion formula of Eq.(3) is asymptotic one with the sign-constant factorially growing high order PT coefficients $t_{n}^{M}$. This means that at some orders of PT the series in (3) start to diverge. Indeed, from the results of direct calculations, performed at the two-loop level in the works [11-13] and at the three-loop level in Refs. $[14,15]$ it is possible to conclude that the perturbative QCD relation between pole and running charm-quark mass diverges from the second (or the third) order of PT. The situation with the mass conversion formula for the $b$-quark is more delicate. Indeed, its PT high-order contributions decrease up to four-loop level, although quite slowly. The four-loop contribution, numerically evaluated in $[17,21]$, is very close to its three-loop term. This allows to affirm that the renormalon nature of the PT series for the $\overline{\mathrm{MS}}$-on-shell mass relation for bottom-quark is manifesting itself from the $O\left(a_{s}^{4}\right)$ contribution. However, in the case of the pole-running top-quark mass relation the perturbative outburst of the corresponding series is not manifesting itself at the four-loop level. In order to understand when the truncated perturbative series can be still used for case of the $t$-quark pole mass, it is necessary to estimate high-order corrections to its relation. This problem is analyzed by us below.

\section{The effective-charges motivated method: from the Euclidean to Minkowski region}

Let us now apply the used in [23] approach for estimations of the high-order PT QCD corrections to the relation between different definitions of heavy quarks. This approach is following the lines of the developed in [24] method of probing the values of high-order perturbative corrections to the renormalization-group invariant quantities, which in its turn is based on the concepts of the effective-charges (ECH) of Ref.[25]. The considered in [23] approach was already used in [26] for estimating four-loop PT QCD corrections to the expression between pole and $\overline{\mathrm{MS}}$ running heavy quark masses, numerically evaluated later on in [17]. As was shown in Refs.[23, 24], from general grounds it is more theoretically justified to use the ECHmotivated procedure to the physical quantities, defined in the Euclidean space-time region, 
and then, if necessary, to translate the expressions of the corresponding PT corrections to the Minkowskian region. This leads to the appearance of the proportional to powers of $\pi^{2}$-terms in the coefficients of the PT series, which relate the quantities, defined in the Minkowskian region. In our case these quantities are the pole and running heavy quark masses.

To clarify how this procedure is working we start from the following formal dispersion representation for the pole masses of the heavy quarks, first considered in [23]:

$$
M_{q}=\frac{1}{2 \pi i} \int_{-\bar{m}_{q}\left(\bar{m}_{q}^{2}\right)-i \epsilon}^{-\bar{m}_{q}\left(\bar{m}_{q}^{2}\right)+i \epsilon} d s^{\prime} \int_{0}^{\infty} \frac{T(s)}{\left(s+s^{\prime}\right)^{2}} d s .
$$

The function $T(s)$ has the meaning of the spectral density and it is defined as $T(s)=$ $\bar{m}_{q}(s) \sum_{n=0}^{\infty} t_{n}^{M} a_{s}^{n}(s)$, where coefficients $t_{n}^{M}$ coincide with the ones, which enter Eqs.(3) and (4). The expression (5) is similar to relation between the determined in the Euclidean region the $e^{+} e^{-}$annihilation Adler function and the Minkowskian time-like ratio $R(s)$, which is one of the main characteristics of the $e^{+} e^{-}$annihilation to hadrons process. The used in Ref.[23] dispersion relation of (5) leads to the conclusion that the analogue of the Adler function, namely the Euclidean quantity $F\left(Q^{2}\right)$ may be defined as

$$
F\left(Q^{2}\right)=Q^{2} \int_{0}^{\infty} d s \frac{T(s)}{\left(s+Q^{2}\right)^{2}} .
$$

Within PT QCD it is expressed through the following series

$$
F\left(Q^{2}\right)=\bar{m}_{q}\left(Q^{2}\right) \sum_{n=0}^{\infty} f_{n}^{E} a_{s}^{n}\left(Q^{2}\right) .
$$

Taking into account the scale dependence of the $\overline{\mathrm{MS}}$-scheme coupling constant and of the running heavy quark masses and using expansions (5-7), we can fix the relations between the coefficients of the Minkowskian series $t_{n}^{M}$ and the determined in the the Euclidean region terms $f_{n}^{E}$ as:

$$
f_{n}^{E}=t_{n}^{M}+\Delta_{n}
$$

In accordance with equation (6) $\Delta_{n}$-contributions contain the proportional to $\pi^{2}$ effects of the analytical continuation. Their detailed derivation at the six-loop level is given in [22]. Here we present the numerical expressions of these contributions for the case of $S U(3)$ color gauge group only. They have the following form:

$$
\begin{aligned}
\Delta_{0} & =0, \quad \Delta_{1}=0, \\
\Delta_{2} & =5.89434-0.274156 n_{l}, \\
\Delta_{3} & =105.6221-10.04477 n_{l}+0.198002 n_{l}^{2}, \\
\Delta_{4} & =2272.002-403.9489 n_{l}+20.67673 n_{l}^{2}-0.315898 n_{l}^{3}, \\
\Delta_{5} & =56304.639-13767.2725 n_{l}+1137.17794 n_{l}^{2}-37.745285 n_{l}^{3}+0.427523 n_{l}^{4}, \\
\Delta_{6} & =1633115.62 \pm 347.65+(-518511.694 \pm 56.723) n_{l}+(61128.1666 \pm 4.7791) n_{l}^{2} \\
& +(-3345.0818 \pm 0.1371) n_{l}^{3}+85.37937 n_{l}^{4}-0.818446 n_{l}^{5} .
\end{aligned}
$$

The analytical expressions for $\Delta_{0}-\Delta_{4}$-terms can be found in $[23,26]$, whereas the analytical expressions for $\Delta_{5}$ and $\Delta_{6}$ were obtained in [22]. The uncertainties, which enter in the numerical expression for $\Delta_{6}$-term are determined by the corresponding inaccuracies of the four-loop 
numerical $t_{41}^{M}$ and $t_{40}^{M}$-contributions to Eq.(4), fixed in [22] by the LS method. Using Eqs.(9) one can find that the numerical values of the $\Delta_{n}$-terms increase considerably with the growth of the order $n$ of PT.

At the next stage of application of the ECH-motivated approach we fix the effective charge $a_{s}^{e f f}\left(Q^{2}\right)$ for the introduced in (7) Euclidean quantity $F\left(Q^{2}\right) / \bar{m}_{q}\left(Q^{2}\right)$

$$
\frac{F\left(Q^{2}\right)}{\bar{m}_{q}\left(Q^{2}\right)}=f_{0}^{E}+f_{1}^{E} a_{s}^{e f f}\left(Q^{2}\right), \quad a_{s}^{e f f}\left(Q^{2}\right)=a_{s}\left(Q^{2}\right)+\sum_{n=2}^{\infty} \phi_{n} a_{s}^{n}\left(Q^{2}\right)
$$

where $\phi_{n}=f_{n}^{E} / f_{1}^{E}$. The coefficients of the ECH $\beta$-function, which is defined as $\beta^{\text {eff }}\left(a_{s}^{\text {eff }}\right)=$ $-\sum_{n \geq 0} \beta_{n}^{\text {eff }}\left(a_{s}^{\text {eff }}\right)^{n+2}$, are related to the coefficients $\beta_{n}$ determined in the $\overline{\mathrm{MS}}$-scheme $\beta$ function of Eq.(2) by the following renormalization-scheme invariant equations:

$$
\begin{aligned}
\beta_{0}^{\text {eff }} & =\beta_{0}, \quad \beta_{1}^{e f f}=\beta_{1}, \quad \beta_{2}^{e f f}=\beta_{2}-\phi_{2} \beta_{1}+\left(\phi_{3}-\phi_{2}^{2}\right) \beta_{0}, \\
\beta_{3}^{\text {eff }} & =\beta_{3}-2 \phi_{2} \beta_{2}+\phi_{2}^{2} \beta_{1}+\left(2 \phi_{4}-6 \phi_{2} \phi_{3}+4 \phi_{2}^{3}\right) \beta_{0}, \\
\beta_{4}^{\text {eff }} & =\beta_{4}-3 \phi_{2} \beta_{3}+\left(4 \phi_{2}^{2}-\phi_{3}\right) \beta_{2}+\left(\phi_{4}-2 \phi_{2} \phi_{3}\right) \beta_{1} \\
& +\left(3 \phi_{5}-12 \phi_{2} \phi_{4}-5 \phi_{3}^{2}+28 \phi_{2}^{2} \phi_{3}-14 \phi_{2}^{4}\right) \beta_{0}, \\
\beta_{5}^{\text {eff }} & =\beta_{5}-4 \phi_{2} \beta_{4}+\left(8 \phi_{2}^{2}-2 \phi_{3}\right) \beta_{3}+\left(4 \phi_{2} \phi_{3}-8 \phi_{2}^{3}\right) \beta_{2} \\
& +\left(2 \phi_{5}-8 \phi_{2} \phi_{4}+16 \phi_{2}^{2} \phi_{3}-3 \phi_{3}^{2}-6 \phi_{2}^{4}\right) \beta_{1} \\
& +\left(4 \phi_{6}-20 \phi_{2} \phi_{5}-16 \phi_{3} \phi_{4}+48 \phi_{2} \phi_{3}^{2}-120 \phi_{2}^{3} \phi_{3}+56 \phi_{2}^{2} \phi_{4}+48 \phi_{2}^{5}\right) \beta_{0} .
\end{aligned}
$$

The starting point of the ECH-motivated estimating procedure of Ref.[24] is the ansatz $\beta_{n}^{\text {eff }}=$ $\beta_{n}$, which should be applied separately at each order of PT beginning from the three-loop one. In the case of the QCD relation between different definitions of heavy quark masses it was used at the three-loop level in Refs.[23, 26] and allowed to get the estimates for the coefficients $\phi_{3}=f_{3}^{E} / f_{1}^{E}$ from Eq.(11a). Further application of the relation (8) with the given in Eq.(9) numerical expressions for the typical to the Minkowski region term $\Delta_{3}$ leads to good agreement of the obtained in $[23,26]$ approximate expression for the $t_{3}^{M}$-coefficient (we denote it as $t_{3}^{M, E C H}$ ) with the explicit three-loop result, obtained in $[14,15]$. It turned out later that the estimated in Ref.[26] by the similar way values of the coefficient $t_{4}^{M, E C H}$ at $n_{l}=3,4,5$ are also in reasonable agreement with the results of the numerical calculations, performed in [17] (see the work of Ref.[21] as well).

These facts serve a-posteriori arguments in favor of the applicability of this ECH-inspired method, supplemented with the explicit expressions for the proportional to $\pi^{2}$ effects of analytical continuation, at higher orders of PT as well. In Ref.[22], which is summarized in brief here, we applied the conditions $\beta_{4}^{e f f}=\beta_{4}$ and $\beta_{5}^{e f f}=\beta_{5}$ for Eqs.(11c) and (11c) and got the numerical estimates for the coefficients $t_{5}^{M, E C H}$ and $t_{6}^{M, E C H}$ after taking into account the analytical continuation contributions $\Delta_{5}$ and $\Delta_{6}$. The concrete numerical results are presented and discussed in Sec.5. Here we only note, that while getting the estimate for the coefficient $t_{6}^{M}$ from Eqs.(11d) and (8) in addition to the theoretical ansatz $\beta_{5}^{e f f}=\beta_{5}$, which of course has definite unfixed theoretical uncertainties, the obtained by the ECH-motivated method at the five-loop level estimate for $f_{5}^{E}$-coefficient was used. Its application at the six-loop order leads to the additional theoretical ambiguity of the estimated value of the $O\left(a_{s}^{6}\right)$-correction to the pole-running heavy quark masses relation, which is not possible to fix. However, in order to study whether there may be extra theoretical ambiguities in the results of applications of the widely spreaded IRR approach, recently used in [27] to analyze the uncertainties of the asymptotic QCD predictions for the coefficients of heavy quark masses relation, we 
will compare in Sec.5 its outcomes with the five and six-loop estimates of the same terms, obtained by means not related to the IRR-approach ECH-inspired methods.

\section{The effective-charges motivated method: direct application in the Minkowski region}

Since the pole masses of heavy quarks are defined in the Minkowski region, it is also worth to consider the predictions of the coefficients in the pole-running heavy quark mass relation applying the ECH-motivated approach of [24] in the time-like region directly. This was first done in [23] (see [26] as well) constructing the Minkowskian analogs of Eqs.(10-11b) for the spectral function of Eq.(5)

$$
T(s)=\bar{m}_{q}(s) \sum_{n=0}^{\infty} t_{n}^{M} a_{s}^{n}(s) .
$$

Defining the ECH $a_{s}^{\text {eff }}(s)$ for the quantity $T(s) / \bar{m}_{q}(s)$ and the corresponding $\tilde{\beta}^{\text {eff }}\left(a_{s}^{\text {eff }}\right)$ function with coefficients $\tilde{\beta_{n}}{ }^{e f f}$, fixed by the replacements $\phi_{n} \rightarrow \phi_{n}^{M}=t_{n}^{M} / t_{1}^{M}$ in Eqs.(10$11 \mathrm{~d})$, one can get the estimates for coefficients $t_{n}^{M, E C H ~ d i r e c t}$ of pole-running heavy quark mass relation directly in the Minkowskian region after using the ansatz $\tilde{\beta}_{n}^{\text {eff }}=\beta_{n}$ at $n \geq 2$. In Ref.[26] it was noticed that the numerical expressions of the third and fourth coefficients $t_{3}^{M, E C H}$ direct and $t_{4}^{M, E C H ~ d i r e c t}$ of the relations between pole and running charm, bottom and top-quark masses are in satisfactory agreement with the values of $t_{3}^{M, E C H}$ and $t_{4}^{M, E C H}$-terms, obtained as described above in Sec.2 (for the detailed comparison see [22]). The estimated five- and six-loop terms, obtained in the Minkowski region directly, have the following form

$$
\begin{aligned}
t_{5}^{M, E C H} \text { direct } & \approx \frac{1}{3 \beta_{0}\left(t_{1}^{M}\right)^{3}}\left[3 t_{2}^{M}\left(t_{1}^{M}\right)^{3} \beta_{3}+t_{3}^{M}\left(t_{1}^{M}\right)^{3} \beta_{2}-4\left(t_{2}^{M} t_{1}^{M}\right)^{2} \beta_{2}\right. \\
& +2 t_{3}^{M} t_{2}^{M}\left(t_{1}^{M}\right)^{2} \beta_{1}-t_{4}^{M}\left(t_{1}^{M}\right)^{3} \beta_{1}+12 t_{4}^{M} t_{2}^{M}\left(t_{1}^{M}\right)^{2} \beta_{0}+5\left(t_{3}^{M} t_{1}^{M}\right)^{2} \beta_{0} \\
& \left.+14\left(t_{2}^{M}\right)^{4} \beta_{0}-28 t_{3}^{M}\left(t_{2}^{M}\right)^{2} t_{1}^{M} \beta_{0}\right] \\
t_{6}^{M, E C H} \text { direct } & \approx \frac{1}{12 \beta_{0}^{2}\left(t_{1}^{M}\right)^{4}}\left[48 t_{4}^{M} t_{3}^{M}\left(t_{1}^{M}\right)^{3} \beta_{0}^{2}+72 t_{4}^{M}\left(t_{1}^{M} t_{2}^{M}\right)^{2} \beta_{0}^{2}+12 t_{2}^{M}\left(t_{1}^{M}\right)^{4} \beta_{0} \beta_{4}\right. \\
& +136\left(t_{2}^{M}\right)^{5} \beta_{0}^{2}-200 t_{3}^{M} t_{1}^{M}\left(t_{2}^{M}\right)^{3} \beta_{0}^{2}-20 t_{4}^{M} t_{2}^{M}\left(t_{1}^{M}\right)^{3} \beta_{0} \beta_{1}-\left(t_{1}^{M}\right)^{3}\left(t_{3}^{M}\right)^{2} \beta_{0} \beta_{1} \\
& +48 t_{3}^{M}\left(t_{1}^{M} t_{2}^{M}\right)^{2} \beta_{0} \beta_{1}-10 t_{1}^{M}\left(t_{2}^{M}\right)^{4} \beta_{0} \beta_{1}-44 t_{2}^{M}\left(t_{1}^{M} t_{3}^{M}\right)^{2} \beta_{0}^{2}+6 t_{3}^{M}\left(t_{1}^{M}\right)^{4} \beta_{0} \beta_{3} \\
& +36\left(t_{1}^{M}\right)^{3}\left(t_{2}^{M}\right)^{2} \beta_{0} \beta_{3}-56\left(t_{1}^{M}\right)^{2}\left(t_{2}^{M}\right)^{3} \beta_{0} \beta_{2}+2 t_{4}^{M}\left(t_{1}^{M}\right)^{4} \beta_{1}^{2}-4 t_{3}^{M} t_{2}^{M}\left(t_{1}^{M}\right)^{3} \beta_{1}^{2} \\
& \left.+8 t_{3}^{M} t_{2}^{M}\left(t_{1}^{M}\right)^{3} \beta_{0} \beta_{2}-6 t_{2}^{M}\left(t_{1}^{M}\right)^{4} \beta_{1} \beta_{3}-2 t_{3}^{M}\left(t_{1}^{M}\right)^{4} \beta_{1} \beta_{2}+8\left(t_{1}^{M}\right)^{3}\left(t_{2}^{M}\right)^{2} \beta_{1} \beta_{2}\right]
\end{aligned}
$$

and like the two-, three- and four-loop analogs can be expressed as

$$
t_{n}^{M, E C H \text { direct }}=f_{n}^{E}-\tilde{\Delta}_{n}
$$

The made in Refs.[22, 26] observations that $t_{3}^{M, E C H \text { direct }} \approx t_{3}^{M, E C H}$ and $t_{4}^{M, E C H \text { direct }} \approx t_{4}^{M, E C H}$ mean that $\Delta_{3} \approx \tilde{\Delta}_{3}$ and $\Delta_{4} \approx \tilde{\Delta}_{4}$, where terms in the l.h.s. are the exactly calculable analytical continuation effects. The presented in Sec.5 comparison of five- and six-loop estimates, obtained within both approaches, demonstrates that the above mentioned approximate equality remains true at the 5 and 6-loops level as well. This demonstrates compatibility of the estimates for heavy quark mass relations coefficients, obtained by applying ECH-inspired procedure both in the Euclidean and Minkowskian regions. 


\section{The estimates by the IRR-based approach}

The most wide-spreaded modern approach of the analysis of high-order PT QCD corrections to physical quantities is based on application of the renormalon technique (for the previous developments see e.g. Refs.[5-9], [28-30]). It is related in part to large $\beta_{0}$-expansion (for the application of the latter one see e.g. $[7,8,29,30])$. As was already mentioned above the asymptotic structure of the PT QCD expression of the pole heavy quark masses through the $\overline{\mathrm{MS}}$-scheme running ones is governed by the leading IRR contribution $[4,5]$, which makes the coefficients of this relation growing factorially with the increase of order of PT. Therefore it is important to analyze the region of applicability of the corresponding asymptotic PT series. For this aim we consider the IRR-based formula [6], which predicts the following factorial behavior of the coefficients $t_{n}^{M}$ :

$$
t_{n}^{M, r-n} \stackrel{n \rightarrow \infty}{\longrightarrow} \pi N_{m}\left(2 \beta_{0}\right)^{n-1} \frac{\Gamma(n+b)}{\Gamma(1+b)}\left(1+\sum_{k=1}^{3} \frac{s_{k}}{(n+b-1) \ldots(n+b-k)}+O\left(n^{-4}\right)\right),
$$

where $\Gamma(x)$ is the Euler Gamma-function, $b=\beta_{1} /\left(2 \beta_{0}^{2}\right)$ and the values of the sub-leading coefficients $s_{k}$ can be found in [27, 31]. Note that our notations and normalizations differ from those introduced in Refs.[5, 6, 27]. In the presented below discussions the coefficients of the RG $\beta$-function depend on $\left(n_{l}-1\right)$ numbers of flavors.

The normalization factor $N_{m}$ in Eq.(15) is the function of $n_{l}$ and of the order $n$ of PT. Unfortunately, its explicit form is not known. Moreover, the way of fixation of $N_{m}$-values is different in various works on the subject (see e.g. [32-34] and the detailed work [31]). This fact introduces the important uncertainty in the IRR-based analysis. In our analysis we use the given in Table 1 numerical results for $n_{l}$-dependence of $N_{m}$, obtained in the process of four-loop analysis of Ref.[27] ${ }^{1}$ :

Table 1. The $n_{l}$ dependence of $N_{m}$ at the the fourth order of PT.

\begin{tabular}{ccccccc}
\hline$n_{l}$ & 3 & 4 & 5 & 6 & 7 & 8 \\
\hline$N_{m}$ & 0.54 & 0.51 & 0.46 & 0.39 & 0.28 & 0.06 \\
\hline
\end{tabular}

In the next section we will study whether the application of this $n_{l}$-dependent value of $N_{m}$ is allowing to get IRR-based estimates, which agree with the five- and six-loop coefficients $t_{5}^{M}$ and $t_{6}^{M}$, evaluated within both Euclidean and Minkowskian ECH-motivated approaches, and respect the following from the large $\beta_{0}$-expansion sign-alternating behavior of their representation through powers of $n_{l}$. In order to find the answer to this problem we should estimate the expressions for $t_{5}^{M}$ and $t_{6}^{M}$-coefficients not only for the physical numbers of light flavors $n_{l}=3,4,5$, which corresponds to the cases of consideration of the charm, bottom at top-quark masses, but for unphysical values of "light" flavors $6 \leq n_{l} \leq 8$ as well.

\section{Numerical results and their interpretation}

We now summarize theoretical discussions of Sec.2-Sec.4 by comparing the estimated expressions for the five and six-loop coefficients in the the relation between pole and MS-scheme running heavy quark masses, obtained by the defined in the Euclidean and Minkowskian regions ECH-motivated methods and by the IRR-based asymptotic formula

\footnotetext{
${ }^{1}$ Note that in [22] while applying the asymptotic expression of Eq.(15) for estimates of the five and six-loop corrections to the $\overline{\mathrm{MS}}$-on-shell mass relation for charm, bottom and top-quarks the same approximate average value $N_{m} \approx 0.5$ was fixed, which in fact is too far from the result, obtained in Refs. [31, 32].
} 
of Eq.(15), which is supplemented with the $n_{l}$-dependent value of the normalization factor $N_{m}$. The concrete $n_{l}$-dependent results for the numerical estimates of $t_{5}^{M}$ and $t_{6}^{M}$-contributions to (3), obtained with the help of the discussed above three methods, are given in Table 2.

Table 2. The estimates of $t_{5}^{M}$ and $t_{6}^{M}$-contributions by three considered methods.

\begin{tabular}{lllllll}
\hline$n_{l}$ & $t_{5}^{M, E C H}$ & $t_{5}^{M, E C H d i r e c t}$ & $t_{5}^{M, r-n}$ & $t_{6}^{M, E C H}$ & $t_{6}^{M, E C H d i r e c t}$ & $t_{6}^{M, r-n}$ \\
\hline 3 & 28435 & 26871 & 34048 & 476522 & 437146 & 829993 \\
\hline 4 & 17255 & 17499 & 22781 & 238025 & 255692 & 511245 \\
\hline 5 & 9122 & 10427 & 13882 & 90739 & 133960 & 283902 \\
\hline 6 & 3490 & 5320 & 7466 & 8412 & 57920 & 137256 \\
\hline 7 & -127 & 1871 & 3119 & -29701 & 15798 & 50520 \\
\hline 8 & -2153 & -196 & 344 & -39432 & -2184 & 4747 \\
\hline
\end{tabular}

One can first observe that for the physical values $n_{l}=3,4,5$ the estimates for the coefficients $t_{5}^{M}$ and $t_{6}^{M}$, obtained by two different realizations of the ECH-based technique, are in reasonable agreement ${ }^{2}$. However, it is surprising that the IRR-based approach with taken from [27] four-loop values of $N_{m}$ does not reproduce the supported by large $\beta_{0}$-approximation sign-alternating $n_{l}$-dependence structure [8] of the corresponding estimated expression for $t_{5}^{M}$ and $t_{6}^{M}$-coefficients , obtained within both realizations of the ECH-approach. Indeed, combining the values for $t_{5}^{M}\left(n_{l}\right)$ as given in three first columns of Table 2 with the representation of Eq.(4), we obtain the following expressions:

$$
\begin{aligned}
t_{5}^{M, E C H} & =2.5 n_{l}^{4}-136 n_{l}^{3}+2912 n_{l}^{2}-26976 n_{l}+86620, \\
t_{5}^{M, E C H d i r e c t} & =1.2 n_{l}^{4}-77 n_{l}^{3}+1959 n_{l}^{2}-20445 n_{l}+72557, \\
t_{5}^{M, r-n} & =-22 n_{l}^{4}+416 n_{l}^{3}-1669 n_{l}^{2}-11116 n_{l}+72972 .
\end{aligned}
$$

The similar surprising feature is observed at the six-loop level, namely:

$$
\begin{aligned}
t_{6}^{M, E C H} & =-4.9 n_{l}^{5}+352 n_{l}^{4}-9708 n_{l}^{3}+131176 n_{l}^{2}-855342 n_{l}+2096737 \\
t_{6}^{M, E C H d i r e c t} & =-2.2 n_{l}^{5}+148 n_{l}^{4}-4561 n_{l}^{3}+71653 n_{l}^{2}-538498 n_{l}+1519440 \\
t_{6}^{M, r-n} & =99 n_{l}^{5}-2903 n_{l}^{4}+30109 n_{l}^{3}-99563 n_{l}^{2}-305378 n_{l}+2040263
\end{aligned}
$$

This paradox of application of the IRR asymptotic formula of Eq.(15) is not clear to us.

Let us now consider the asymptotic structure of the relation between pole and running masses of real heavy quarks. One can see from Table 2 that for $n_{l}=3,4,5$ three methods of estimates of high-order corrections give comparable values for the five-loop coefficients, while the six-loop large coefficients, estimated by the ECH-motivated approaches, are lower than the existing asymptotic IRR-renormalon predictions by the factor 2 only. Since for rather approximate estimate we do not consider this difference seriously, we will use these estimated by three methods numbers as inputs of our numerical studies. We fix the values of the the running masses of $c, b$ and $t$-quarks following the presented in [22] considerations as $\bar{m}_{c}\left(\bar{m}_{c}^{2}\right)=1.275 \mathrm{GeV}, \bar{m}_{b}\left(\bar{m}_{b}^{2}\right)=4.180 \mathrm{GeV}, \bar{m}_{t}\left(\bar{m}_{t}^{2}\right)=164.3 \mathrm{GeV}$ and take the following values of the $\overline{\mathrm{MS}}$-scheme strong coupling constant, normalized at these running masses, viz $\alpha_{s}\left(\bar{m}_{c}^{2}\right)=0.3947, \alpha_{s}\left(\bar{m}_{b}^{2}\right)=0.2256, \alpha_{s}\left(\bar{m}_{t}^{2}\right)=0.1085$. Taking into account the known results of direct diagram calculations $[10-15,21]$ and using the data, presented in Table 2, we find

\footnotetext{
${ }^{2}$ It is worth emphasizing that our ECH-inspired results for $t_{5}^{M}$-coefficient at $n_{l}=4$ agree rather well with the approximate value of this term, independently obtained in Ref.[35] as one of the outcomes of the global fits of characteristics of the bottomonium spectrum studied in non-relativistic QCD up to $\mathrm{N}^{3} \mathrm{LO}$.
} 
that within the both ECH-motivated methods and the IRR-based approach the asymptotic PT expressions for the pole masses of charm, bottom and top-quarks has the following form:

$$
\begin{aligned}
\frac{M_{c}}{1 \mathrm{GeV}} \approx 1.275+0.214+0.208+0.295+0.541 \\
+\{\underbrace{1.135+2.389}_{\mathrm{ECH}} ; \underbrace{1.072+2.192}_{\mathrm{ECH} \text { direct }} ; \underbrace{1.359+4.162}_{\mathrm{IRR}, N_{m}=0.54}\}, \\
\frac{M_{b}}{1 \mathrm{GeV}} \approx 4.180+0.400+0.200+0.146+0.137 \\
+\{\underbrace{0.137+0.137}_{\mathrm{ECH}} ; \underbrace{0.140+0.147}_{\mathrm{ECH} \text { direct }} ; \underbrace{0.182+0.293}_{\mathrm{IRR}, N_{m}=0.51}\}, \\
\frac{M_{t}}{1 \mathrm{GeV}} \approx 164.300+7.566+1.614+0.498+0.196 \\
+\{\underbrace{0.074+0.025}_{\mathrm{ECH}} ; \underbrace{0.084+0.037}_{\mathrm{ECH} \text { direct }} ; \underbrace{0.112+0.079}_{\mathrm{IRR}, N_{m}=0.46}\} .
\end{aligned}
$$

Based on these results we conclude that five-loop corrections to pole mass of charm-quark are rather close in all three considered estimate methods. However, the six-loop corrections, predicted with help of the IRR technique, differ significantly from the ones, obtained by the both ECH procedures. With reference to the $b$-quark pole mass the situation is more interesting. Indeed, the ECH-motivated method that takes into account the transition from the Euclidean to Minkowskian regions demonstrates output to some kind of plateau (four, five and six-loop corrections coincide), whereas the direct ECH and the IRR approaches indicate the growth of these corrections. These facts testify to the unconditional manifestation of the asymptoticity of the corresponding PT series for bottom-quark starting with five-loop order. For case of $t$-quark all three considered estimate procedures outline the decrease of the five and six-loop corrections. This means that the asymptotic structure of this PT series is not yet manifesting itself at these levels. Therefore the conception of pole mass of top-quark can be safely used even at the $O\left(a_{s}^{6}\right)$ level.

\section{Conclusion}

We apply three approximate methods for estimation of the five and six-loop corrections to the $\overline{\mathrm{MS}}$-on-shell heavy quark mass relation, namely two ECH-motivated methods, defined in the Euclidean and Minkowskian regions correspondingly, and the infrared renormalon based approach. By means of these methods we determine flavor dependence of the considered contributions in the $O\left(a_{s}^{5}\right)$ and $O\left(a_{s}^{6}\right)$ orders. Wherein the IRR-based technique with normalization $N_{m}$-factor, taken in the four-loop approximation, does not give questionable $n_{l}$-dependent results while the both ECH approaches predict not only close values of the corresponding coefficients but reproduce the sign-alternating structure of these corrections in expansion in powers of massless flavors. The numerical studies of all estimate procedures indicate the growth of the five and six-loop corrections to the pole mass of charm-quark. Whereas the ECH Euclidean method for $b$-quark pole mass leads to effect of plateau and the rest two methods outline also the increase of these corrections. In the case of $t$-quark the asymptotic nature of the corresponding PT series is not observed even at six-loop level. Therefore the concept of the pole mass of top-quark is applicable up to 6 order of PT for sure. 


\section{Acknowledgments}

This contribution is based on the talks presented by one of us (V.M.) at the XXth International Seminar on High Energy Physics "Quarks-2018" (27 May - 2 June, Valday) and at the VII International Workshop "Calculation for Modern and Future Colliders (CALC2018)" (22 July-1 August, JINR, Dubna). We would like to thank organizers of both these events for creating inspiring atmosphere for scientific discussions. The authors wish to thank K.G. Chetyrkin, L. Dudko and S. Moch for useful discussions at "Quarks-2018" Seminar and A. G. Grozin for valuable comments, made after the talk at CALC-2018 Workshop, We are also grateful to V.M.Braun for hellpful discussions. The work of A.K. is supported in part by the Russian Science Foundation grant No. 14-22-00161, while the work of V.M. and his stay in Valday was supported by the Russian Science Foundation grant No. 16-12-10151.

\section{References}

[1] P. A. Baikov, K. G. Chetyrkin and J. H. Kühn, Phys. Rev. Lett. 118, no. 8, 082002 (2017) [arXiv:1606.08659 [hep-ph]].

[2] F. Herzog, B. Ruijl, T. Ueda, J. A. M. Vermaseren and A. Vogt, JHEP 1702, 090 (2017) [arXiv:1701.01404 [hep-ph]].

[3] P. A. Baikov, K. G. Chetyrkin and J. H. Kühn, 'JHEP 1410, 076 (2014) [arXiv:1402.6611 [hep-ph]].

[4] I. I. Y. Bigi, M. A. Shifman, N. G. Uraltsev and A. I. Vainshtein, ' Phys. Rev. D 50, 223 (1994) [hep-ph/9402360].

[5] M. Beneke and V. M. Braun, Nucl. Phys. B 426, 301 (1994) [hep-ph/9402364].

[6] M. Beneke, Phys. Lett. B 344, 341 (1995) [hep-ph/9408380].

[7] M. Beneke and V. M. Braun, Phys. Lett. B 348, 513 (1995) [hep-ph/9411229].

[8] P. Ball, M. Beneke and V. M. Braun, Nucl. Phys. B 452, 563 (1995) [hep-ph/9502300].

[9] M. Beneke,' Phys. Rept. 317, 1 (1999) [hep-ph/9807443].

[10] R. Tarrach, Nucl. Phys. B 183, 384 (1981) .

[11] N. Gray, D. J. Broadhurst, W. Grafe and K. Schilcher, Z. Phys. C 48, 673 (1990).

[12] L. V. Avdeev and M. Y. Kalmykov, Nucl. Phys. B 502, 419 (1997) [hep-ph/9701308].

[13] J. Fleischer, F. Jegerlehner, O. V. Tarasov and O. L. Veretin, Nucl. Phys. B 539, 671 (1999) Erratum: [Nucl. Phys. B 571, 511 (2000)] [hep-ph/9803493].

[14] K. Melnikov and T. v. Ritbergen, Phys. Lett. B 482, 99 (2000) [hep-ph/9912391].

[15] K. G. Chetyrkin and M. Steinhauser, Nucl. Phys. B 573, 617 (2000) [hep-ph/9911434].

[16] R. Lee, P. Marquard, A. V. Smirnov, V. A. Smirnov and M. Steinhauser, JHEP 1303, 162 (2013) [arXiv:1301.6481 [hep-ph]].

[17] P. Marquard, A. V. Smirnov, V. A. Smirnov and M. Steinhauser, Phys. Rev. Lett. 114, no. 14, 142002 (2015) [arXiv:1502.01030 [hep-ph]].

[18] Y. Kiyo, G. Mishima and Y. Sumino, JHEP 1511, 084 (2015) [arXiv:1506.06542 [hep$\mathrm{ph}]$.

[19] A. L. Kataev and V. S. Molokoedov, Eur. Phys. J. Plus 131, no. 8, 271 (2016) [arXiv:1511.06898 [hep-ph]].

[20] A. L. Kataev and V. S. Molokoedov, J. Phys. Conf. Ser. 762 no.1, 012078 (2016) [arXiv:1604.03485 [hep-ph]].

[21] P. Marquard, A. V. Smirnov, V. A. Smirnov, M. Steinhauser and D. Wellmann, Phys. Rev. D 94, no. 7, 074025 (2016) [arXiv:1606.06754 [hep-ph]]. 
[22] A. L. Kataev and V. S. Molokoedov, "Multiloop contributions to the $\overline{\mathrm{MS}}$-on-shell mass relation for heavy quarks in QCD and charged leptons in QED and the asymptotic structure of the perturbative QCD series", ' INR-TH-2018-15, arXiv:1807.05406 [hep-ph].

[23] K. G. Chetyrkin, B. A. Kniehl and A. Sirlin, Phys. Lett. B 402, 359 (1997) [hep$\mathrm{ph} / 9703226]$.

[24] A. L. Kataev and V. V. Starshenko, Mod. Phys. Lett. A 10, 235 (1995) [hep$\mathrm{ph} / 9502348]$.

[25] G. Grunberg, Phys. Rev. D 29, 2315 (1984)

[26] A. L. Kataev and V. T. Kim, Phys. Part. Nucl. 41, 946 (2010) [arXiv:1001.4207 [hep$\mathrm{ph}]]$.

[27] M. Beneke, P. Marquard, P. Nason and M. Steinhauser, Phys. Lett. B 775, 63 (2017) [arXiv:1605.03609 [hep-ph]].

[28] M. Beneke and V. I. Zakharov, Phys. Rev. Lett. 692472 (1992).

[29] D. J. Broadhurst, A. L. Kataev and C. J. Maxwell, Nucl. Phys. B 592, 247 (2001) [hep$\mathrm{ph} / 0007152]$.

[30] A. L. Kataev, "Light quark masses and renormalons"; CERN-TH-2001-030; hepph/0102223; in Proc. of 11th International Seminar Quarks-2000, Pushkin, Russia, May 13-21, 2000; Eds. G.B. Pivovarov, V.A. Matveev and V.A. Rubakov ; INR Press; Moscow 2002, pp.35-52.

[31] A. Pineda, JHEP 0106, 022 (2001) [hep-ph/0105008].

[32] F. Campanario, A. G. Grozin and T. Mannel, Nucl. Phys. B 663, 280 (2003) Erratum: [Nucl. Phys. B 670 (2003) 331], [hep-ph/0303052].

[33] C. Ayala, G. Cvetič and A. Pineda, JHEP 1409045 (2014) 045 [arXiv:1407.2128 [hep$\mathrm{ph}]]$.

[34] C. Ayala, G. Cvetic and A. Pineda, J. Phys. Conf. Ser. 762 no.1, 012063 (2016) [arXiv:1606.01741 [hep-ph]].

[35] V. Mateu and P. G. Ortega, JHEP 1801, 122 (2018) [arXiv:1711.05755 [hep-ph]]. 